\title{
A Modeling Method of Cloud-based Cyber-Physical Systems
}

\author{
Qiuxia Yang a) and Lichen Zhang ${ }^{\text {b) }}$ \\ Faculty of Computer Science and Technology Guangdong University of Technology Guangzhou 510090, China \\ a) Qiuxia Yang: yqx1415@163.com \\ b)LichenZhang@zhanglichen1962.com
}

\begin{abstract}
The emerging Cyber Physical Systems (CPSs) are a kind of novel intelligent complex systems that tightly integrated different scales of computation and physical components and interacted under the future network. A cloud computing environment offers a simplified, centralized platform or resources for use when needed at a low cost. In this paper, we propose a MDA modeling method for Cloud-based CPS. Through building on the top of a extension of the UML modeling language to model Cyber Physical Systems based the Cloud Platform. We illustrate the proposed approach using an example of Rail Control System.
\end{abstract}

Key words: Cyber physical systems; model; cloud; uml.

\section{INTRODUCTION}

Cyber Physical Systems (CPSs)[1] [2] are integrations of monitoring, communication and computation operation. Cyber Physical System is a safety-critical system which have strict safety, real-time and reliability requirements. The quality of CPSs is very important as their failure may case loss of life or property. Nowadays, CPSs are widely used in the traffic control systems, high confidence medical systems, process control systems, advanced automotive systems, avionics systems, defense systems, distributed robotics, critical infrastructure control systems, manufacturing and so on.

The Model-driven Architecture (MDA)[3] is a model driven development framework. MDA has been used to improve the quality of software and to increase the productivity. MDA has been used for model-driven development of models, where the MDA methods improve the quality of models. The Unified Modeling Language(UML[4]) and the Meta it is beli Object Facility(MOF[5]) has become a popular choice among software engineers. The UML and the MOF provide native extension techniques, and the examples include UML profiles[6], metamodel slicing[7], pruning/reduction, or package referencing and merging.

Cloud computing[8] can offer the IT resource capabilities as a service and provide an effective solution for addressing some problems arising from CPSs. The could computing infrastructure can effectively support the collection, storage and processing of a large number of distributed heterogeneous data. Meanwhile, cloud computing can provide a unified platform that can support CPSs requirements, including the hierarchical structure, information management, and security management etc. Science the support of the cloud platform and cloud service application, CPSs will promote the efficiency of the system, increase the quality of service, and improve the friendliness of environment, particularly in a big data environment.

The big data technology and cloud computing are now widely using CPSs. The traditional modeling approach has been unable meet new require in the cloud environment. There is a big challenge for cloud-based CPS modeling method. In this paper, we propose the MDA modeling method base on extend UML for cloud-based CPSs. The remainder of this paper is organized as follows: The section 2 presents model-driven modeling method of cloudbased cyber physical systems. Section 3 presents the case study with the proposed method. Finally, we present some conclusions. 


\section{MODEL-DRIVEN MODELING METHOD OF CLOUD-BASED CYBER-PHYSICAL SYSTEM}

UML is a modeling language which supports text and graphics. UML provides a extension mechanism by UML profile. But UML profile is not an UML meta model and is an important part of UML extension mechanism. UML profile is mainly composed of stereotype, constraints and tagged value, and which is based on UML and MOF [5]. Stereotype can add new things or metamodel for UML. Constraints mainly constrains the elements in the model semantically. However, Object Constraint Language(OCL) provides a standard way to express the constraints of UML2.0 model effectively.

The paper proposes a Cloud-based CPS UML (CCUML) which is a software as services extension for UML based cloud platform CPS. The language considers cloud services as an extension concept of UML profile called CCUML profile, as shown in Fig.1.

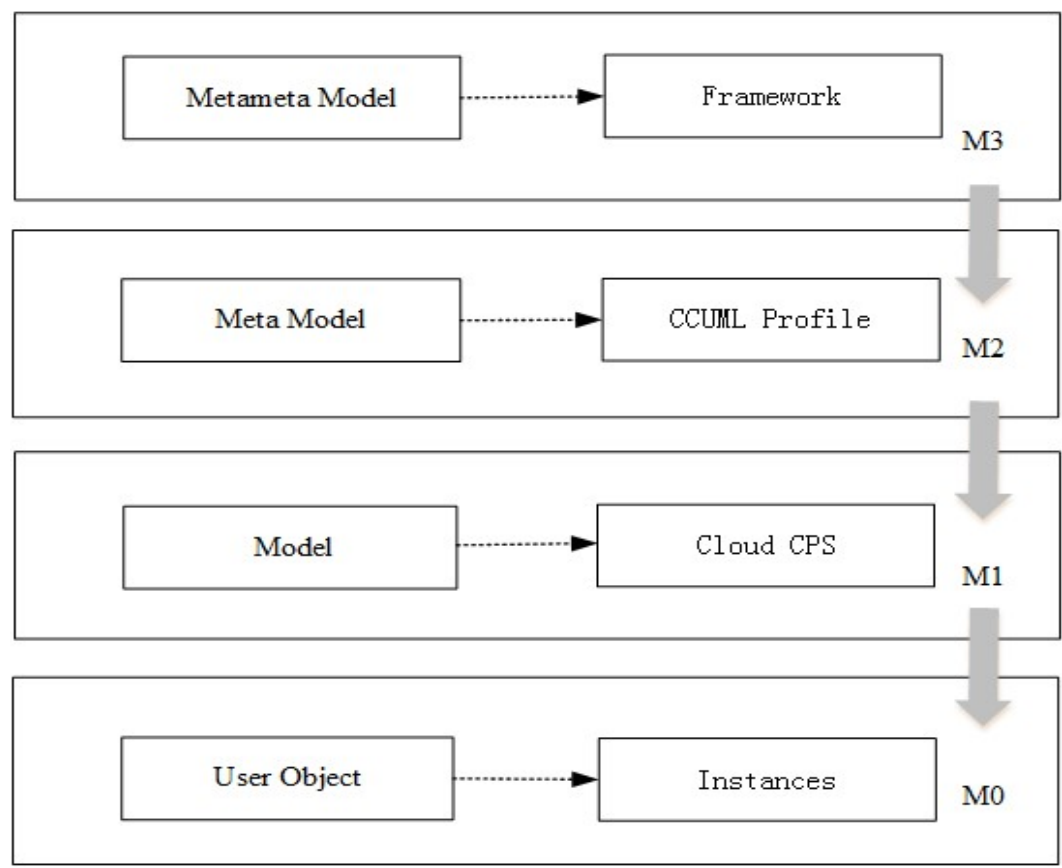

FIG.1 CCUML Model Driven Architecture

The CCUML unifies and generalizes previous object-oriented modeling language UML. By cloud computing, the subsystem of the CPS can exist independently as a service and share it on the cloud platform.

By marking the corresponding stereotype, the model elements are used to the corresponding primitives. The stereotype $<<$ Users $>>$ represents system user. The stereotype $<<$ Provider $>>$ represents an abstract service provider, which refers to the the complete member in the registration process. The stereotype $<<$ CloudServie $>>$ represents services coming from the services suppliers which can provide the corresponding function modules according to their needs. The stereotype $<<$ CloudManagement $>>$ represents an management of service operation including service composition and clasification etc. The stereotype $<<$ NonFunctionAttribute $>>$ represents a non-functional property of services. The stereotype $<<$ CloudEnvironment $>>$ represents services environment which is from the virtual dynamic resource pool to the user or system as required provide storage capacity,computing capacity. The stereotype $<<$ Image $>>$ represents the mirror of cloud environment. The CCUML profile is described by IBM rational software architect (RSA) modeling tool, and the metamodel of CCUML is shown in Figure. 2. 


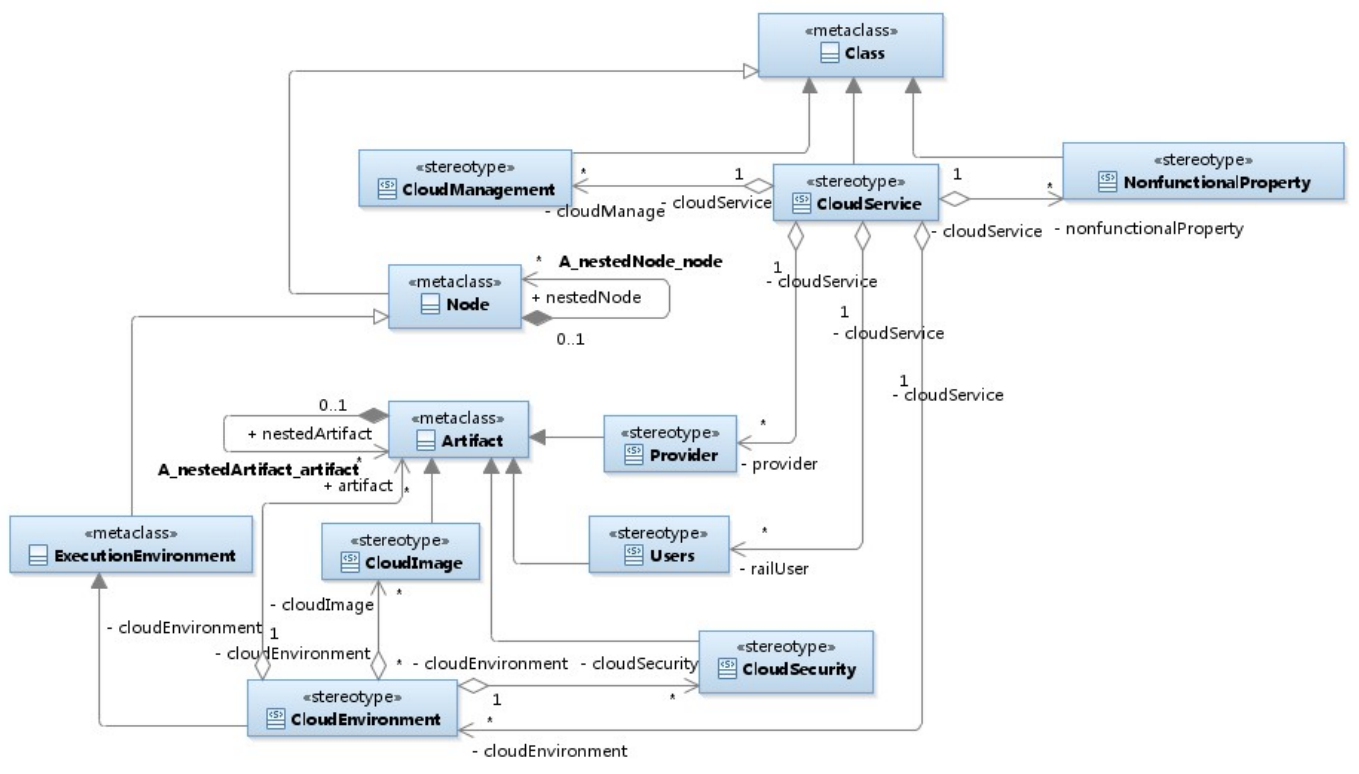

FIG.2 CCUML Metamodel

\section{CASE STUDY}

Railway control system is a typical cyber physical system. The big data technology [9] and cloud computing are widely using railway control system. The development of cloud-based railway control system has become an inevitable trend. So, we used cloud-based railway control system as an example to model. Railway control system can be effectively split into four subsystems as shown in Fig.3. Railway control system is including data communication subsystem(DCS), automatic train supervision subsystem(ATS), zone control subsystem (ZCS) and vehicle on-board subsystem(VOBS) [10]. Through the data communication subsystem, the ATS(ATP) subsystem is obtained separately the information of database, wayside devices, train position and movement authority from the permissions of the database storage unit in the data communication subsystem, vehicle on-board subsystem and zone control subsystem by the data communication subsystem.

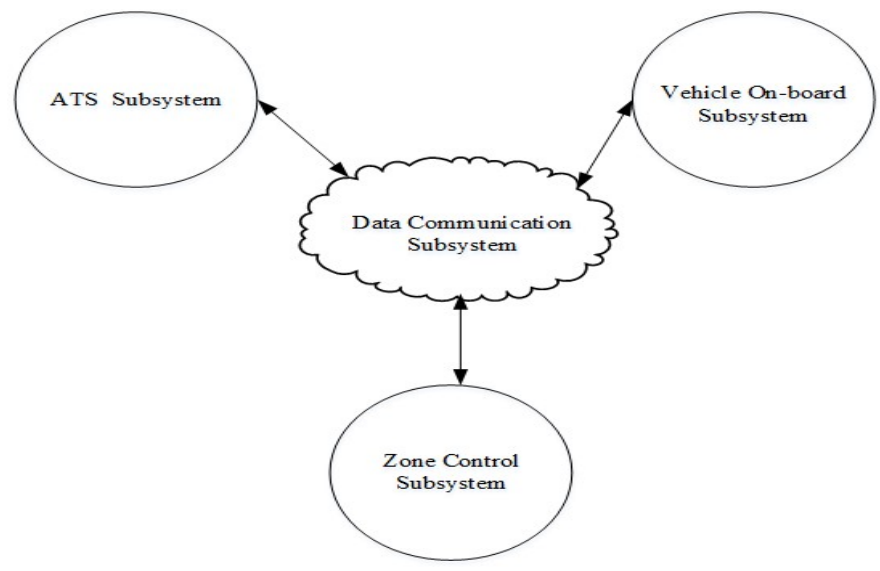

FIG.3 The Communication Between the Subsystems

We use CCUML to model the system model of the rail cyber physical systems, and CBTC system's model is described by IBM rational software architect (RSA) modeling tool, the system model is shown in Fig.4. 


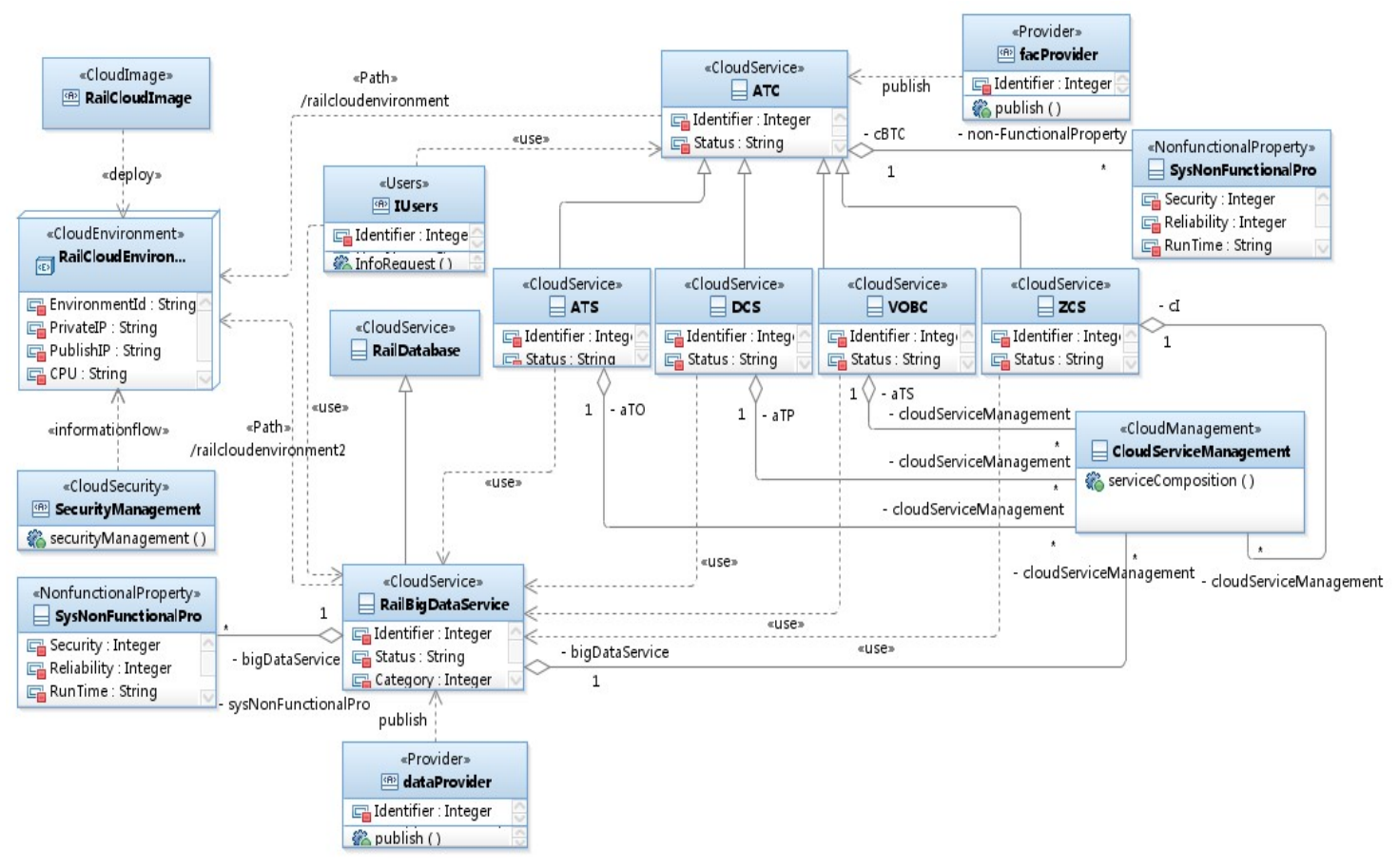

FIG.4 The CBTC System Model

\section{CONCLUSION}

In this paper, we proposed an approach to model cloud-based cyber physical systems based on model-driven method. We illustrated the proposed CCUML approach by the communication-based train control system to demonstrate that can be for modeling complex cloud-based cyber physical systems.

The further work is devoted to developing tools to support the automatic generation of model.

\section{REFERENCES}

1. E. A. Lee, "CPS foundations,” in Proc. 47th Des. Autom. Conf., New York,NY, USA: ACM, 2010, pp. 737742.

2. Herkersdorf, "Multicore enablement for Cyber Physical Systems", 2012 International Conference on Embedded Computer Systems(SAMOS),2012, pp. 345

3. G. Casale, et al. "Dice: Quality-driven development of dataintensive cloud applications", Proceedings of the 7th International Workshop on Modeling in Software Engineering (MiSE 2015), 2015

4. International Workshop on Modeling in Software Engineering (MiSE 2015), 2015 Object Management Group, OMG Unified Modeling Language (OMG UML), 2015a, (available at: http://www.omg.org/spec/UML). Version 2.5, formal/2015-03-01.

5. Object Management Group, OMG Meta Object Facility (MOF) core specification, 2015b, (available at: http://www.omg.org/spec/MOF). Version 2.5, formal/2015-06-05.

6. G. Giachetti, B. Marín, O. Pastor, "Using UML as a domain-specific modeling language: A proposal for automatic generation of UML profiles" in Proceedings of the 21 st International Conference on Advanced Information Systems Engineering, LNCS, 5565, Springer, 2009, pp. 110-124

7. A. Blouin, B. Combemale, B. Baudry, O. Beaudoux, "Kompren: Modeling and generating model slicers", Softw. Syst. Model,14 (1),2015, pp.321-337.

8. Kuyoro S. O.; Ibikunle F; \& Awodele O., “Cloud Computing Security Issues and challenges"' in Proceeding of International Journal of Computer Networks (IJCN), Volume (3), Issue (5), 2011. 
9. Dunren Che, Mejdl Safran, Zhiyong Peng,'From Big Data to Big Data Mining: Challenges, Issues, and Opportunities".HoloMAS 2013, LNAI 8062, 2013, pp. 305-316.

10. Chan-Ho Cho,Dong-Hyuk Choi,Zhong-Hua Quan,Sun-Ah Choi,"GieSoo Park and Myung-Seon Ryou. Modeling of CBTC Carborne ATO Functions using SCADE".11th International Conference on Control, Automation and Systems,2011, pp.1089-1094. 